\title{
Analog-to-digital conversion of information in the retina
}

\author{
Andrey N. Volobuev, Eugeny. S. Petrov
}

Samara State Medical University, Samara, Russia; volobuev@,samaramail.ru

Received 17 November 2010; revised 19 December 2010; accepted 22 December 2010.

\begin{abstract}
We considered the physiological mechanisms of functioning of the retina's neural network. It is marked that the primary function of a neural network is an analog-to-digital conversion of the receptor potential of photoreceptor into the pulse-to-digital signal to ganglion cells. We showed the role of different types of neurons in the work of analog-to-digital converter. We gave the equivalent circuit of this converter. We researched the mechanism of the numeric coding of the receptor potential of the photoreceptor.
\end{abstract}

Keywords: Analog-to-Digital Converter; A Ganglion Cell; Oscillator of Clock Frequency; Pulse; Intensity; Neuron; Action Potential; the Retina; Photoreceptor; Digital-to-Analog Converter

\section{INTRODUCTION}

Conversion of information in the retina is carried out both by the photoreceptor and by the brachiate network of neurons. Initial perception of the light pulse occurs in the retinal photoreceptors - in rods and cones. Rods are responsible for the black and white, twilight vision. They are evenly distributed on the nervous tunic (the retina) of eyeball, their total number is about 100 million units in each retina [1]. Cones, which are 3 million units, are responsible for the color and daytime vision. They are more concentrated in the central part (fovea centralis) of the retina $\left(140000\right.$ cones on $\left.\mathrm{mm}^{2}\right)$.

Feature of the photoreceptors is the hyperpolarizing nature of the receptor potential which is generated in response to light stimulation. Figure 1 shows the time dependence of the receptor potential $U_{\text {in }}$ of single cones that arises in response to short flashes of the light $(10 \mathrm{~ms})$ by three different light intensities [2]. The potential $U_{\text {in }}$ subsequently proceed to the input of neural network of retina.

In the darkness on the photoreceptor membrane resting potential of a little more (modulo) value of $-20 \mathrm{mV}$ (see Figure 1). Curve 1 corresponds to the weakest in- tensity $I_{1}$, curve 2 - the light intensity $I_{2}=4 I_{1}$, curve 3 - the intensity $I_{3}=16 I_{1}$. Between the intensity of the light flash and the receptor potential analog (logarithmic) dependence was observed.

\section{THE EQUIVALENT ELECTRIC CIRCUIT OF THE NEURAL NETWORK RETINA}

Receptor potential $U_{\text {in }}$ photoreceptor through synaptic switching proceeds at the input of a bipolar neuron. At Figure 2 the distribution of electrical signals along the system retinal neurons is shown. Circuit of the neural network adopted in accordance with [3], a slight simplification of concern, in particular, the amount involved in information processing similar amacrine neurons. These simplifications we will discuss further.

Neural network retina represents a slightly modified circuit of the analog-to-digital converter (ADC) consistent accounts [4]. Block diagram of the ADC, the corresponding neural network retina is shown in Figure 3. Each element of the ADC converts the analog signal from the photoreceptor PR into digital code, corresponds to a neuron. In particular, the comparator $\mathrm{K}$ corresponds

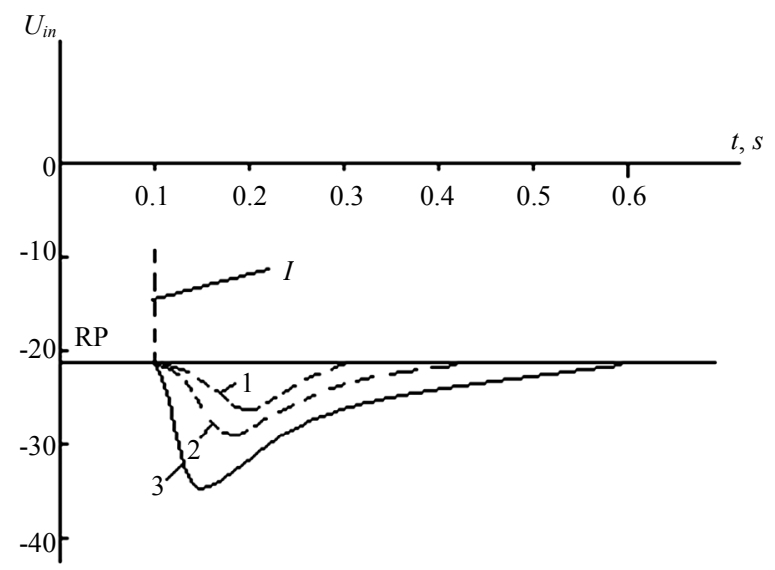

Figure 1. Time dependence of receptor potential of the rod by its stimulation with a light flash: RP - the resting potential, I moment of light flash, $U_{\text {in }}$-receptor potential in $\mathrm{mV}$ at the input into the neural network of the retina, $t$ - time in seconds. 


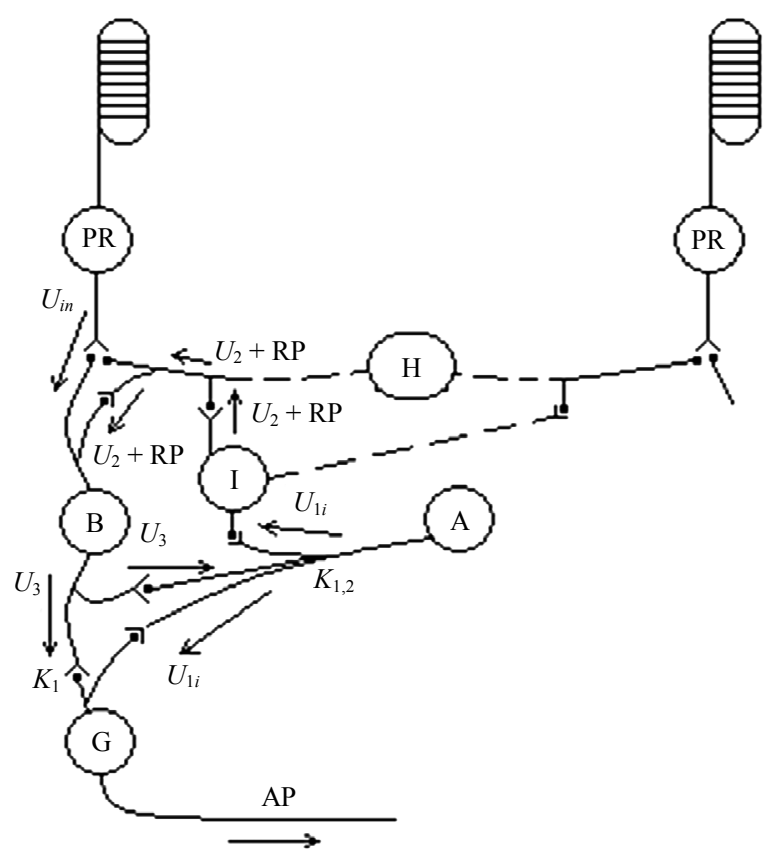

Figure 2. Schematic circuit of the neural network retina. Showing photoreceptor PR and the following neurons: B-bipolar, A-amacrine, I-interplexiform, G-ganglionic, $\mathrm{H}$ - horizontal. $U_{2}+\mathrm{RP}$ - analog output potential of the I; $U_{1 \mathrm{i}}$ - pulse voltage A; $U_{3}$-analog output voltage of the $\mathrm{B} ; \mathrm{K}_{1}$, $\mathrm{K}_{2}$ - places the signal is interrupted (keys); AP - the action potential. The dotted line shows the version of the signal passage from the I through the body of horizontal neuron $\mathrm{H}$.

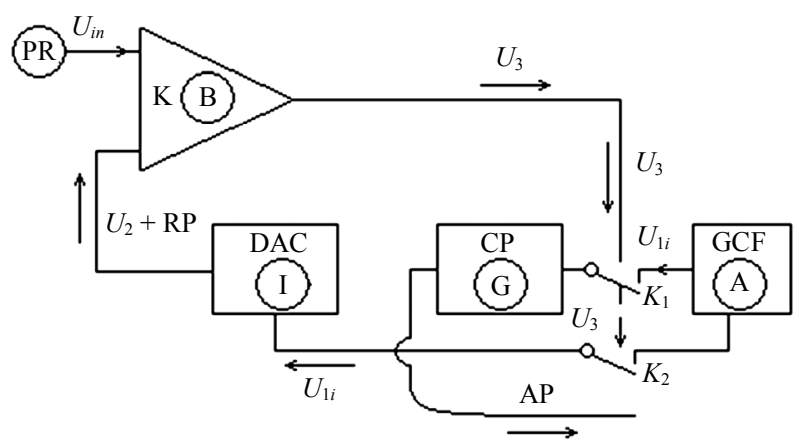

Figure 3. Equivalent circuit of a neural network retina: $K_{1}, K_{2}$ -keys.

diaxon B, digital-to-analog converter (DAC) - interplexiform neuron I, pulse counter (PC) - ganglionic neuron $\mathrm{G}$, generator clock frequency $\mathrm{GCF}$ - amacrine neuron A. Action potentials AP originally generated by amacrine neurons and in the sequel, according to the used encoding, through the excitation of ganglionic neurons are transmitted to fibers of the optic nerve.

Horizontal neurons apparently feebly involved in the analog-to-digital conversion. Their primary function is providing contrast boundaries in the field of view visual image [1]. Analysis of this neural network of the retina problem in this article is not performed.

The purpose of this study is to examine the transition of hyperpolarizing receptor potential of photoreceptors in the pulse sequence of action potentials of ganglion neurons.

Let us consider in more detail the equivalent circuit of the ADC retina, shown in Figure 3. Showing items are required for any variety of $\mathrm{ADC}$. One of the most important elements is the generator of clock frequency (GCF), which generates a continuous sequence of pulses, in the simplest case-with a constant frequency. This sequence of electrical pulses $U_{1 \mathrm{i}}$ is shown in Figure $\mathbf{4 a}$.

It is known that amacrine neurons generate action potentials [3], but until now their role is not clear [5]. There are identified by morphological and histochemical methods about 30 species amacrine neurons [1]. There's also stated that amacrine cells "react to the inclusion and turning off lights, which simply signaling about change of lighting, regardless of its direction." This correlates well with their role GCF in the circuit ADC. A large variety of amacrine neurons indicates about variability parameters of ADC retina.

An important element of the ADC is the comparator $\mathrm{K}$. It does not work with pulsed signals, and compares the two analog signals - the input to the neural network of photoreceptor $U_{\text {in }}$ and the output signal from digital-toanalog converter (DAC), gradually and step increases to

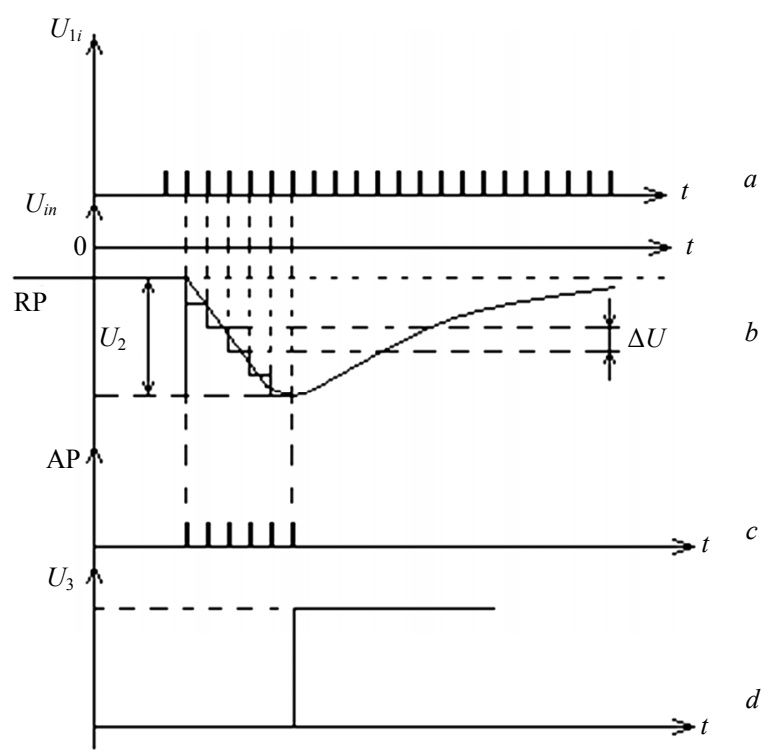

Figure 4. Graphs of the analog-to-digital conversion of receptor potential in the retina: $a$ - a sequence of AP amacrine neurons, $b$ - the accumulation with a step $\Delta U$ of potential comparison $U_{2}+\mathrm{RP}$ interplexiform neuron, $\mathrm{RP}$ - the resting potential of photoreceptors, $c-$ a countable sequence (spike) AP of ganglion neurons; $d$ - the output signal of bipolar neurons. 
$U_{2}+\mathrm{RP}$ (see Figure 3). In case of equality $U_{\text {in }}=U_{2}+$ RP comparator generates a constant voltage to the $U_{3}$, see Figure 4d. This is consistent with the fact that bipolar neurons do not generate action potentials. Signal for bipolar neurons spreads like electrotonic [2].

DAC is a required element of ADC. It is discrete, with a step $\Delta U$ (Figure $4 \boldsymbol{b}$ ), accumulates potential $U_{2}+\mathrm{RP}$ at its output for comparison with the receptor potential $U_{\text {in }}$ photoreceptor (see Figure 3).

An interplexiform neuron feedback plays the role of DAC. On the input of this neuron by its impulse branches served $U_{1 \mathrm{i}}$ impulse voltage and on the output occurs a step analog potential comparison $U_{2}+$ RP. So far the role and functioning of these neurons were not described. Some authors [1] consider that the role of these neurons is not very important and call them inter-retinal.

Pulse counter in the ADC retina-is undoubtedly the ganglionic neuron G (see Figures 2,3). Ganglionic neurons direct the signal in the form of action potentials to the central nervous system.

\section{INFORMATION CODING BY A NEURAL NETWORK RETINA}

Work ADC retina begins from supply the receptor potential $U_{\text {in }}$ produced by the photoreceptors to the input of the comparator $\mathrm{K}$. By the closed keys $\mathrm{K}_{1}$ and $\mathrm{K}_{2}$ (see Figure 3) the impulses from the GCF served on the pulse counter, where they come out in the form of a sequence of action potentials, and on the DAC, where the potential comparison accumulate $U_{2}+R P$ (Figure $4 \boldsymbol{b}$ ). If the conditions arise $U_{i n}=U_{2}+\mathrm{RP}$ comparator generates a voltage of $U_{3}$ (Figure $4 d$ ), which breaks the outputs from the GCF by keys $\mathrm{K}_{1}$ and $\mathrm{K}_{2}$. It is also possible to supply the signal $U_{3}$, produced by the comparator $\mathrm{K}$ (bipolar neurons), directly on ganglionic neurons $\mathrm{G}$ (pulse counter PC) to stop the generation of pulses of that neuron. Synaptic switching from the B on the G is another analog of the key $\mathrm{K}_{1}$ in Figure 2. Thus, each signal from the photoreceptor, ganglionic neuron (or pulse counter) generates a strictly defined number of pulses $n$-pulsed digital signal.

Consequently, the neural network retina carries numeric coding of the analog signal at the output from a photoreceptor with the help of a certain quantity of pulses. In this case, the quantity of pulses generated by the ganglion neurons is proportional to the amplitude of the analog receptor potential, $n \sim U_{i n}$.

If we consider that the duration of the signal rise $U_{i n}$ (modulo) of approximately $0.1 \mathrm{~s}$ (see Figure 1) and the duration of the action potential of ganglionic neuron is 1 $\mathrm{ms}$ (the frequency of pulsation in the optic nerve comes up to 1000 pulses per second [6]), then to one receptor potential of photoreceptor a ganglionic neuron generates a spike, consisting of approximately 100 action potentials. Figure $4 c$ shows the conditional six pulses.

It is noteworthy that the signal passage $U_{2}+\mathrm{RP}$ along the horizontal branches of a neuron $\mathrm{H}$ (see Figure 2). Some bipolar neurons B (comparators) receive a signal comparison $U_{2}+\mathrm{RP}$ from DAC (interplexiform neurons) not directly but through the body of horizontal neurons [3] (see Figure 2). This fact indicates the involvement of horizontal neurons in work of the ADC of the retina, or vice versa, the involvement of the ADC in the work of horizontal neurons, there is a mutual influence of the analog-to-digital conversion in the retina and the formation process of the contrast of the visual image. The involvement of interplexiform neurons is in work of horizontal neurons also noted in [1]. It is possible that the signal, which passage from the I through the branches of body $\mathrm{H}$, need (or energetically favorable) to add the stepwise changing of the voltage $U_{2}$ to resting potential $\mathrm{H}$. However, the cause of the signal passage from the I through the branches or body $\mathrm{H}$ requires further detailed investigation.

The task of providing information about contrast boundaries in the total amount of information sent to the central nervous system is crucial. Early H. Helmholtz pointed out the imperfections of the optical system of the eye, and as a consequence, the poor quality of the image on the retina [7]. Formation of a contrast boundary in the field of view carry out by submitting a brake signal from the horizontal neurons to bipolar neurons, in case, if the photoreceptors, that are connected with these bipolar neurons, provide a weak receptor potential. In this case, approximate and strongly illuminated photoreceptors can generate a large receptor potential and the brake signal from the horizontal neurons doesn't send on the bipolar neurons, connected with them. The necessity for information that weak receptor signals should not be involved in the generation of potential comparison by interplexiform neurons may also be a cause of the signal passage from the I through the branches or body $\mathrm{H}$.

It should be noted that the circuit of the neural network retina [3] is somewhat more complicated than that is shown in Figure 2. In particular, the generator of clock frequency GCF as an amacrine cells A is duplicate, apparently, to give to ADC a greater reliability. There is a feedback of pulse branch of interplxsiform (DAC) and amacrine neurons (GCF) which is not shown in Figures 2,3. Perhaps it is necessary to stop the pulsation of GCF by achieving equality $U_{\text {in }}=U_{2}+\mathrm{RP}$, since otherwise, by continuous work of GCF happen depletion amacrine neurons. Perhaps this branch of interplexiform neuron provides a more rigid circular connection of two duplicated amacrine neurons in the retinal neural network. 


\section{CONCLUSIONS}

Encoding an analog signal at the output from a photoreceptor to a digital code is apparently one of the main functions of the neural network retina. Retinal neurons perform well-defined functions: a comparison of analog signals, the generation of clock frequency, digital-toanalog conversion, counting pulses. At the same time, horizontal neurons in the retina carry out other important tasks, such as staining of the visual image. Horizontal neurons are involved in the work of an analog-to-digital converter retina.

Fulfilled analysis indicates the importance of stimulating the development of electropulse physiotherapy procedures for diseases of the neural network retina.

\section{REFERENCES}

[1] Guyton, A.K. and Hall, D.E. (2008) Medical physiology. Logosphere, Moscow.

[2] Samoilov, V.O. (2004) Medical Biophysics. SpetsLit, S-Peterburg.

[3] Kamkin, A.G. and Kamensky, A.A. (2004) Fundamental and Clinical Physiology. Academia, Moscow.

[4] Pryanishnikov, V.A. (2009) Electronics: A complete course of lectures. Corona-Century, S-Peterburg.

[5] Hubel, D. (1990) Eye, Brain, Vision. Mir, Moscow.

[6] Kositsky, G.I. (1985) Human Physiology. Medicine, Moscow.

[7] Schmidt, R. and Thews, G. (1996) Human Physiology. Mir, Moscow. 